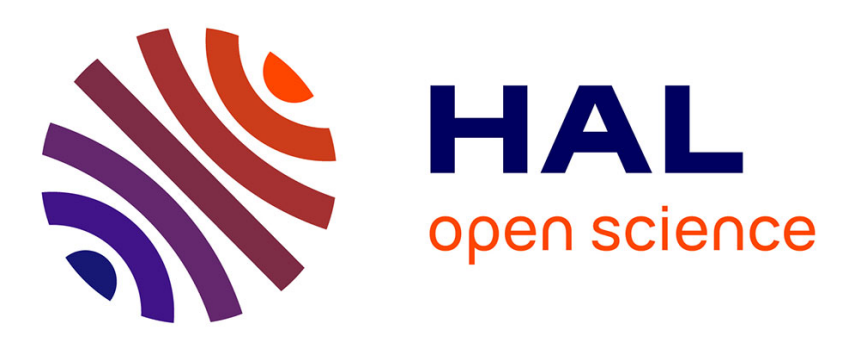

\title{
A clique-covering sufficient condition for hamiltonicity of graphs
}

\author{
Alain Bretto, Thierry Vallée
}

\section{To cite this version:}

Alain Bretto, Thierry Vallée. A clique-covering sufficient condition for hamiltonicity of graphs. Information Processing Letters, 2009, 109 (20), pp.1156-1160. 10.1016/j.ipl.2009.07.015 . hal-01010265

\section{HAL Id: hal-01010265 \\ https://hal.science/hal-01010265}

Submitted on 19 Jun 2014

HAL is a multi-disciplinary open access archive for the deposit and dissemination of scientific research documents, whether they are published or not. The documents may come from teaching and research institutions in France or abroad, or from public or private research centers.
L'archive ouverte pluridisciplinaire HAL, est destinée au dépôt et à la diffusion de documents scientifiques de niveau recherche, publiés ou non, émanant des établissements d'enseignement et de recherche français ou étrangers, des laboratoires publics ou privés. 


\title{
A clique-covering sufficient condition for hamiltonicity of graphs
}

\author{
Alain Bretto $^{\mathrm{a}, *}$, Thierry Vallée ${ }^{\mathrm{b}}$ \\ a Université de Caen, GREYC-CNRS UMR 6072, Bd Maréchal Juin, F14032 Caen Cedex, France \\ ${ }^{\mathrm{b}}$ Mathematical Sciences Dept., Georgia Southern University, Statesboro, 30458 GA, United States
}

\begin{abstract}
A B S T R A C T
A graph is hamiltonian if it contains a cycle which goes through all vertices exactly once. Determining if a graph is hamiltonian is known as NP-complete problem and no satisfactory characterization for hamiltonian graphs has been found. There are several necessary conditions for hamiltonicity and since the seminal work of Dirac in 1952 , many sufficient conditions were found. These conditions are usually expressed in terms of node degree, connectivity, density, toughness, independent sets, regularity and forbidden subgraphs. In this article we give an extended clique decomposition condition ensuring the hamiltonicity of a large class of graphs. Then we discuss briefly the possibility of broader extensions as well as algorithmic issues.
\end{abstract}

\section{Introduction and preliminaries}

Since the results of Dirac in [3], many sufficient conditions for hamiltonicity of graphs have been found, and a huge literature exists on the subject surveyed for instance in $[6,2,1,4]$. The hamiltonicity problem and related topics are still topical and the search continues for sufficient conditions [7].

Most of the existing sufficient conditions for hamiltonicity are expressed in terms of node degree, connectivity, density, toughness, independent sets, regularity and forbidden subgraphs of the graph.

In addition to a condition expressed in terms of 2-connectivity and forbidden subgraphs, S. Goodman and S. Hedetniemi introduced in [5] two other conditions based only on the existence of what is called here a clique-covering of the graph. A clique-covering is a decomposition of the graph into complete subgraphs (cliques). The two sufficient conditions are expressed in terms of the existence of clique-coverings satisfying two different sets of additional properties.
In this paper we focus our attention on the more interesting of the two conditions. We generalize this condition to prove the hamiltonicity of a broader class of graphs. In the conclusion we discuss the possibility of broader extensions as well as the possibility to design an efficient algorithm, able to find a clique-covering satisfying the above mentioned properties iff such a covering exists.

In the sequel, we use the usual set theory notations. In particular $|X|$ denotes the size or cardinal of $X$, and $X \backslash Y$ the complement of the set $Y$ in $X$. We also define $\mathbb{P}(X)$ as the set of all pairs $\{x, y\}$ of elements of $X$ such that $x$ and $y$ are distinct.

We always suppose a graph to be undirected, simple (no loop) and finite. Thus we define a graph as a pair $(V, E)$, where $V$ is a set of vertices and $E \subseteq \mathbb{P}(V)$ is a set of edges. Despite the fact that we are interested in hamiltonicity of simple graphs, our main result is proved via a construction involving multigraphs (cf. Definition 6 and Theorem 1). We use the following formalization of multigraphs.

Definition 1. A multigraph is a triplet $G=(V, E, \varepsilon)$ where:

- $V$ is the set of vertices of $G$.

- $E$ is the set of edges of $G$.

\footnotetext{
* Corresponding author.

E-mail addresses: alain.bretto@info.unicaen.fr (A. Bretto), vallee_th@yahoo.fr (T. Vallée).
} 
- $\varepsilon: E \mapsto \mathbb{P}(V)$ is a function which associates every $e \in$ $E$ to a pair $\{x, y\}$ of distinct vertices. The vertices $x, y$ are said incident to $e$.

It is sometimes useful to denote by $V(G)$ (resp. $E(G)$ ) the set of vertices (resp. set of edges) of a graph or multigraph $G$. A walk in a graph or multigraph is an alternative sequence of vertices and edges such that the vertices on the left and right of the edge are incident to the edge and distinct. Notice that a walk may include several times the same vertex or edge. For a graph $G$, we write $x \smile_{G} y$ to express that $\{x, y\} \in E(G)$ or simply $x \smile y$ if there is no ambiguity on $G$. Similarly, we write $x=x_{0} \smile x_{1} \smile \ldots \smile$ $x_{k}=y$ to denote a walk of length $k$ in the graph. A walk is a closed walk if moreover $x=y$. A closed walk is a cycle if it contains no repetition of vertex except $x=y$.

A path is a walk containing no repetition of vertex (and so no repetition of edge). A path (resp. cycle) is hamilton if it contains every vertex of the graph or multigraph. A graph or multigraph is hamiltonian if it contains a hamilton cycle. It is eulerian if it contains a cycle containing each edge exactly once.

A graph or multigraph is connected if all pairs of distinct vertices $x, y$ are connected by a walk, and complete if every such a pair is connected by an edge.

Note that, according to the definitions above, a vertex is considered as a cycle of length 0 , and thus the singleton graph $(\{x\}, \varnothing)$ and the singleton multigraph $(\{x\}, \emptyset, \varnothing)$ are connected, complete, eulerian and hamiltonian.

We write $H \subseteq G$ to express that $H$ is a subgraph of $G$. The subgraph induced by a set of vertices of $G$ is defined as usual. The (open) neighborhood is defined by:

$N(x)=\{y:\{x, y\} \in E\}$

and the closed neighborhood by:

$N[x]=\{x\} \cup N(x)$.

A clique $C$ of a graph $G$ is a set $C \subseteq V(G)$ such that the subgraph induced by $C$ is complete. Notice that a clique is not necessarily maximal. By a slight abuse, we sometimes identify a clique $C$ with its induced graph. Notice that $N(x)$ is a clique iff $N[x]$ is a maximal one.

We introduce now the notion of neighborhood equivalence among nodes:

Definition 2. Let $G$ be a graph, we define the equivalence relation $\equiv$ on the vertices of $G$ by, for all $x, y \in V(G)$ :

$x \equiv y \quad \Leftrightarrow \quad G[x]=G[y]$.

We denote by $\bar{x}$ the equivalence class of $x$ modulo $\equiv$, and we say that $x, y$ are neighborhood equivalent if $x \equiv y$.

Notice that $\bar{x}$ forms a clique. Moreover if $x$ belongs to a clique $C$ and $y \in \bar{x}$ then every $z \in C$ is adjacent to $y$ (since $G[x]=G[y])$. Consequently we have:

Fact 1. For every graph $G$ and $x \in C \subseteq V(G)$ : $C$ is a clique iff $C \cup \bar{x}$ is a clique.
A vertex $x$ of a graph $G$ is simplicial if $N(x)$ is a clique or equivalently if $N[x]$ is a maximal clique of $G$. We denote by $S(G)$ the set of simplicial vertices of $G$ and by $N S(G)$ the set of non-simplicial vertices of $G$.

We can characterize neighborhood equivalence among simplicial vertices as follows.

Proposition 1. For all $x \in S(G)$ and $y \in V(G)$, we have:

$y \in \bar{x} \quad \Leftrightarrow \quad y \in N[x] \cap S(G)$.

Proof. If $y \in \bar{x}$ we have $N[x]=N[y]$ and $N[x]$ being a maximal clique clearly $y \in N[x] \cap S(G)$. Conversely, assume that $y \in N[x] \cap S(G)$. Because $y \in N[x], y$ is adjacent to $x$. Because $N[y]$ is a clique for every $z \in N[y], z$ is adjacent to $x$, so $N[y] \subseteq N[x]$. Because $y \in S(G), N[y]=N[x]$ ( $x$ being simplicial).

Lemma 1. Let $G$ be a connected graph. The following statements are equivalent:

1. $G$ is complete.

2. $V(G)=S(G)$.

3. There exists $x \in S(G)$ such that $N[x] \subseteq S(G)$.

Proof. The facts $1 \Rightarrow 2$ and $2 \Rightarrow 3$ are trivial. Thus it remains to show $3 \Rightarrow 1$.

Suppose that 3 is true. From Proposition 1 for all $y \in$ $N[x]$ we have $N[y]=N[x]$, so $G$ being a connected graph we have $G=N[x]$.

\section{Clique-coverings of graphs}

We introduce here the notion of a eulerian cliquecovering of a graph. The next section will show that a connected graph "coverable" with an eulerian clique-covering is hamiltonian. We first define the notion of clique-covering of a graph.

Definition 3. A clique-covering (c-covering) of a graph $G$ is a set $\mathcal{C}$ of cliques of $G$ such that:

1. $C \subseteq C^{\prime} \Rightarrow C=C^{\prime}$, for all $C, C^{\prime} \in \mathcal{C}$.

2. Every edge of $G$ is contained in at least one clique of $\mathcal{C}$.

3. Every vertex of $G$ is contained in at least one clique.

Notice that by the third condition of the definition $\{\{x\}\}$ is a c-covering of the singleton graph $G=(\{x\}, \emptyset)$, it is also clearly the unique one. Moreover, if $G$ is connected and of order at least 2 then every vertex is incident to at least one edge. Thus, in that case, the first and second conditions of the definition imply that every $C \in \mathcal{C}$ is of order at least 2 . The following facts are elementary.

Fact 2. If $\mathcal{C}$ is a c-covering of the graph $G$ then for all $x \in V(G)$ and $C \in \mathcal{C}$ :

1. $x \in C \Rightarrow C \subseteq N[x]$,

2. $N[x]=\bigcup\{C \in \mathcal{C}: x \in C\}$.

3. $|N[x]| \leqslant 2 \Rightarrow N[x] \in \mathcal{C}$. 
Definition 4. Let $\mathcal{C}$ be a c-covering of the graph $G$ and $x$ be a vertex of $G$ :

- $x$ is $\mathcal{C}$-isolated iff it belongs to a unique clique of $\mathcal{C}$.

- $x$ is a $\mathcal{C}$-junction iff it belongs to at least two cliques of $\mathcal{C}$.

We denote by $I(\mathcal{C})($ resp. $J(\mathcal{C}))$ the set of $\mathcal{C}$-isolated vertices of $G$ (resp. $\mathcal{C}$-junctions of $G$ ). We define also $J(C)=$ $J(\mathcal{C}) \cap \mathcal{C}$, for every $\mathcal{C} \in \mathcal{C}$.

Obviously $I(\mathcal{C})$ and $J(\mathcal{C})$ are disjoint and since every vertex is contained in at least one clique of $\mathcal{C}, I(\mathcal{C})$ and $J(\mathcal{C})$ form a partition of $V(G)$. Note also that if the vertex $x$ is isolated in $G$ (that is, if $\{x\}$ is a connected component of $G$ ) then it is $\mathcal{C}$-isolated. The converse is not true in general.

Proposition 2. If $\mathcal{C}$ is a c-covering of $G$, for every $x \in V(G)$, we have $N[x] \in \mathcal{C}$ iff $x$ is $\mathcal{C}$-isolated.

Proof. Assume that $N[x] \in \mathcal{C}$. If $x \in C \in \mathcal{C}$ then $C \subseteq N[x]$ by Fact 2.1 , and so $C=N[x]$ by first condition of Definition 3 . Thus $x$ is $\mathcal{C}$-isolated.

Assume now that $x$ belongs to a unique clique $C \in \mathcal{C}$. We have $C \subseteq N[x]$, and if we suppose $y \in N[x] \backslash C$, the edge $\{x, y\}$ is then covered by a clique $C^{\prime} \in \mathcal{C}$ different from $C$, which is contradictory.

Corollary 1. $I(\mathcal{C}) \subseteq S(G)$ and $N S(G) \subseteq J(\mathcal{C})$, for every c-covering of $G$.

Proof. If $x$ is $\mathcal{C}$-isolated, we have $N[x] \in \mathcal{C}$ by Proposition 2 and thus $N[x]$ is complete, that is, $x \in S(G)$. If now $x \in N S(G)$, there exist $y, z \in N[x]$ which are not adjacent and thus the edges $\{x, y\}$ and $\{x, z\}$ belong to two different cliques of $\mathcal{C}$ and thus $x \in J(\mathcal{C})$.

A natural question arising from Corollary 1 concerns the converse inclusions: Is $S(G) \subseteq I(\mathcal{C})$ and $J(\mathcal{C}) \subseteq N S(G)$ ? The answer to this question is no in general, as it is shown in the example below where the c-covering $\mathcal{C}_{2}$ does not verify $S(G) \subseteq I(\mathcal{C})$

Example 1. The graph $G$ in Fig. 1 contains two simplicial vertices $a, d$ which are both $\mathcal{C}_{1}$-isolated, nevertheless $d$ is not $\mathcal{C}_{2}$-isolated. See also Fig. 2.

It is easy to check that both the c-covering $\mathcal{C}_{1}$ and $\mathcal{C}_{2}$ above are eulerian in the sense given below. It will be shown in the next section (Corollary 2 ) that both the existence of $\mathcal{C}_{1}$ and the existence of $\mathcal{C}_{2}$ can be used to prove the hamiltonicity of the graph $G$.

Definition 5 (Eulerian c-covering). A c-covering $\mathcal{C}$ of a graph $G$ is:

1. pairwise-joint if each vertex is contained in no more than two cliques.

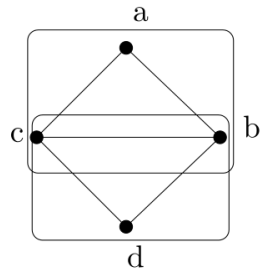

A c-covering $\mathcal{C}_{1}$

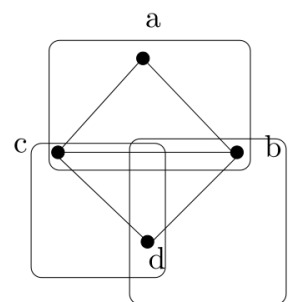

A c-covering $\mathcal{C}_{2}$
Fig. 1. This graph contains two simplicial vertices $a, d$ which are both $\mathcal{C}_{1}$-isolated, but $d$ is not $\mathcal{C}_{2}$-isolated
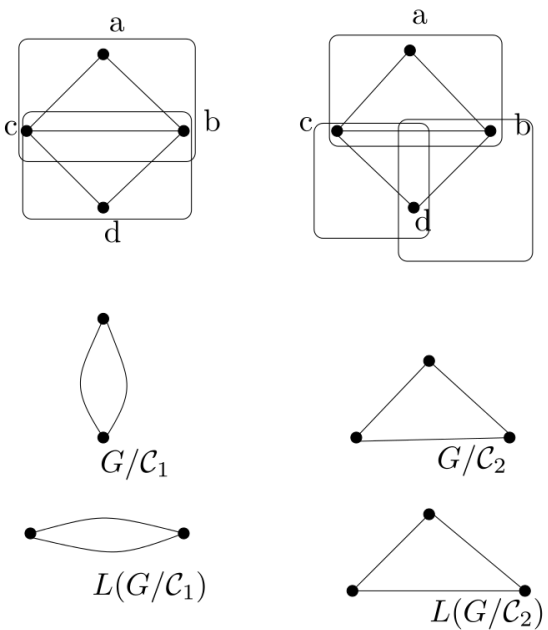

Fig. 2. The graph of Example 1 with two of its coverings $\mathcal{C}_{1}, \mathcal{C}_{2}$, the in duced multigraphs, and the line graphs of these induced multigraphs.

2. evenly-joint if each clique contains an even number of $\mathcal{C}$-junctions.

3. eulerian if it is pairwise-joint and evenly-joint.

Lemma 2. If $\mathcal{C}=\left(C_{u}\right)_{u \in U}$ is a pairwise-joint c-covering of a graph $G$, then for every vertex $x$ the following statements are equivalent:

1. $N[x] \notin \mathcal{C}$.

2. $x \in J(\mathcal{C})$.

3. There exists a unique pair of cliques $C, C^{\prime} \in \mathcal{C}$ such that $x \in$ $C \cap C^{\prime}$ and $N[x]=C \cup C^{\prime}$.

Proof. That $1 \Leftrightarrow 2$ is immediate by Proposition 2 , and that $3 \Rightarrow 2$ is immediate by definition of $J(\mathcal{C})$. Now, if $x \in J(\mathcal{C})$, there exists at least two distinct $C, C^{\prime} \in \mathcal{C}$ such that $x \in$ $C \cap C^{\prime}$. The uniqueness of $C, C^{\prime}$ is then immediate since $\mathcal{C}$ is supposed pairwise-joint and by Fact 2.2 we have $N[x]=$ $C \cup C^{\prime}$.

We recall that $J(C)$ is the set of $\mathcal{C}$-junctions of $C \in \mathcal{C}$, where $\mathcal{C}$ is a c-covering. Clearly, $J(C)=\bigcup\left\{C \cap C^{\prime}: C^{\prime} \in \mathcal{C}\right.$, $C^{\prime} \neq C$. Moreover, when $\mathcal{C}$ is pairwise-joint the following lemma clearly holds. Note that the lemma is not necessary here, it is nevertheless interesting to mention it. 
Lemma 3. Let $\mathcal{C}$ be a pairwise-joint c-covering of a graph $G$. For every $C \in \mathcal{C}$, the family $\left\{C \cap C^{\prime}: C^{\prime} \in \mathcal{C}, C^{\prime} \neq C\right\}$ forms a partition of $J(C)$ and so:

$$
|J(C)|=\sum_{C^{\prime} \in \mathcal{C} \backslash\{\mathcal{C}\}}\left|C \cap C^{\prime}\right| .
$$

\section{Eulerian c-covering and hamiltonicity}

A graph $G$ is eulerian-coverable if there exists an eulerian c-covering of $G$. In this section, we show that every eulerian coverable connected graph is hamiltonian.

Firstly, we associate to every graph $G$ and every pairwise-joint clique covering $\mathcal{C}$ of $G$ a multigraph $G / \mathcal{C}$. Secondly, we prove in Theorem 1 that if $G / \mathcal{C}$ is eulerian then $G$ is hamiltonian.

Definition 6. Let $G$ be a graph and $\mathcal{C}$ a pairwise-joint ccovering of $G$. The multigraph $G / \mathcal{C}$ is defined by:

1. $V(G / \mathcal{C})=\mathcal{C}$.

2. $E(G / \mathcal{C})=J(\mathcal{C})$.

3. $\varepsilon(x)=\left\{C, C^{\prime}\right\}$ iff $x \in C \cap C^{\prime}$, for all $C, C^{\prime} \in \mathcal{C}$ and $x \in$ $J(\mathcal{C})$.

Notice that, since $\mathcal{C}$ is pairwise-joint, every $x \in J(\mathcal{C})$ is contained in a unique pair of cliques $C, C^{\prime}$ (Lemma 2). Thus $G / \mathcal{C}$ is well-defined as a multigraph since each edge is associated by $\varepsilon$ to a unique pair of vertices (cf. Definition 1 ).

Lemma 4. For every pairwise-joint c-covering $\mathcal{C}$ of $G$ : $G$ is connected iff $G / \mathcal{C}$ is.

Proof. Suppose first that $G$ is connected and let $C, C^{\prime}$ be two distinct cliques of $\mathcal{C}$. Since neither $C \subseteq C^{\prime}$ nor $C^{\prime} \subseteq C$, there exists $x \in C \backslash C^{\prime}$ and $y \in C^{\prime} \backslash C$, and there exists a walk $x=x_{0} \smile \cdots \smile x_{k}=y$ in $G$. It is then straightforward to build a walk in $G / \mathcal{C}$ connecting $C$ and $C^{\prime}$, using the fact that $\mathcal{C}$ covers all the edges of $G$.

If we suppose now that $G$ is not connected then $\mathcal{C}$ is clearly the disjoint union of clique-coverings of the connected components of $G$. Moreover, the intersection of two cliques of two different connected components being empty, it is obviously impossible to build a walk in $G / \mathcal{C}$ between two such cliques.

Theorem 1. Let $G$ be a connected graph and $\mathcal{C}$ be a pairwisejoint c-covering of $G$. The first two following statements are equivalent and moreover they imply the third one.
1. $G / C$ is eulerian.
2. $|J(C)|$ is even, for every $C \in \mathcal{C}$.
3. $G$ is hamiltonian.

Proof. Note that $G / \mathcal{C}$ is connected by Lemma 4 . Notice now that for every $C \in \mathcal{C}$ the degree of $C$ in $G / \mathcal{C}$ is clearly equal to the number of $\mathcal{C}$-junctions of $C$. It is well known that a multigraph is eulerian iff the degree of each vertex is even. That proves $1 \Leftrightarrow 2$. We prove now $1 \Rightarrow 3$.
$1 \Rightarrow 3$ Suppose $G / \mathcal{C}$ eulerian. If $\mathcal{C}$ contains a unique clique $C$ and thus $G / \mathcal{C}=(\{C\}, \emptyset, \emptyset)$, then $G$ is complete since $V(G)=C$, and so hamiltonian. Suppose now that $\mathcal{C}$ contains at least two cliques and thus $G / \mathcal{C}$ contains at least one edge. Let $T=C_{0} x_{0} C_{1} \ldots C_{k} x_{k} C_{k+1}$, where $C_{k+1}=C_{0}$, be an eulerian cycle of $G / \mathcal{C}$. By hypothesis, every edge of $G / \mathcal{C}$, that is, every vertex of $J(\mathcal{C})$ appears exactly once in $T$. For every $i \in\{0, \ldots, k\}$, we have $x_{i} \in C_{i} \cap C_{i+1}$ by definition of $G / \mathcal{C}$ and so $x_{i}, x_{i+1} \in C_{i+1}$ (where $x_{k+1}$ is defined as $x_{0}$ ). Thus, since $C_{i+1} \in \mathcal{C}$ is a clique of $G$, it follows that:

- There exists a hamilton path $P_{i+1}$ in the subgraph induced by $C_{i+1} \cap I(\mathcal{C})$ (defined as the empty path if $\left.C_{i+1} \cap I(\mathcal{C})=\emptyset\right)$.

- $x_{i} \smile x_{i+1}$.

- $x_{i} \smile P_{i+1} \smile x_{i+1}$ is a path in $C_{i+1}$ and thus in $G$.

Hence $W=x_{0} \smile P_{1} \smile x_{1} \smile \cdots \smile x_{k} \smile P_{k+1} \smile x_{k+1}$ is a closed walk in $G$. By eulerianity of $T$ each of the $\mathcal{C}$-junctions $x_{0}, \ldots, x_{k}$ appears exactly once in $W$ except for the endpoint $x_{0}=x_{k+1}$. Moreover, since $G / \mathcal{C}$ is connected, every vertex $C \in V(G / \mathcal{C})$ is incident to at least one edge of $G / \mathcal{C}$. Hence $C$ appears at least once in $T$ and is among $C_{1}, \ldots, C_{k+1}$. Thus, every $\mathcal{C}$-isolated vertex of $G$, since it belongs to a clique $C \in \mathcal{C}$, appears at least once in a path of the form $P_{i+1}$ of $W$. Nevertheless it may appear in several of these paths since they are built from the vertices (cliques) of $T$ which may be repeated in $T$. Thus to obtain a hamilton cycle it is sufficient to remove from $W$ the possible repetitions of the $\mathcal{C}$-isolated vertices. It is easily done by replacing the path $x_{i} \smile P_{i+1} \smile x_{i+1}$ by the shortcut $x_{i} \smile x_{i+1}$ whenever there exists $j<i$ such that $C_{j+1}=C_{i+1}(j, i \in\{0, \ldots, k\})$, that is, whenever the $\mathcal{C}$-isolated vertices of $C_{i+1}$ are already contained in $P_{j+1}$ and so in $x_{0} \smile P_{1} \smile \cdots \smile P_{i} \smile x_{i}$.

Corollary 2. If there exists an eulerian c-covering of the connected graph $G$ then $G$ is hamiltonian.

We close this section by showing that the sufficient condition for hamiltonicity of graphs expressed by Corollary 2 is more general than the one given in [5]. First we recall the latter.

Corollary 2a. (See [5].) If $\mathcal{C}$ is a set of cliques of a connected graph $G$ which forms a partition of the edges of $G$, such that every vertex belongs to exactly two cliques, and such that every clique contains an even number of vertices, then $G$ is hamiltonian.

Lemma 5. If a set of cliques of a graph $G$ satisfies the conditions of Corollary $2 a$ then it is an eulerian c-covering of $G$.

Proof. Let $\mathcal{C}$ be a set of cliques of $G$ satisfying the conditions of the corollary. Clearly, since $\mathcal{C}$ forms a partition of $E(G)$, every edge of $G$ is covered by a clique of $\mathcal{C}$, and $C \subseteq C^{\prime}$ implies $C=C^{\prime}$ for all $C, C^{\prime} \in \mathcal{C}$. Since $G$ is connected, every vertex is incident to at least one edge as long as $|V(G)|>1$, and thus $\mathcal{C}$ covers $V(G)$. Hence $\mathcal{C}$ is a c-covering of $G$. Now, since every vertex of $G$ is contained in 


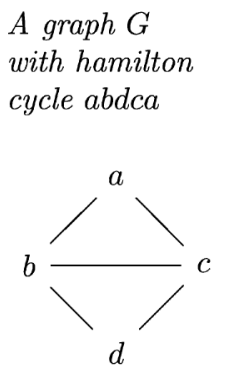

A graph $H$

with hamilton

cycle abedca

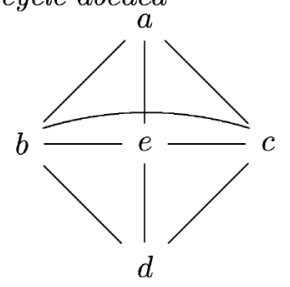

Fig. 3.

exactly two cliques, the c-covering $\mathcal{C}$ is pairwise-joint and moreover every vertex of $G$ is a $\mathcal{C}$-junction. Thus for every $C \in \mathcal{C}$ the number of $\mathcal{C}$-junctions is exactly the order of $C$ which is even by hypothesis. Thus $\mathcal{C}$ is an eulerian c-covering.

The graph $G$ of Example 1, recalled in Fig. 3, is an instance of a graph which can be proved hamiltonian using Corollary 2 but not using Corollary 2a. Indeed, as already mentioned, it can be proved hamiltonian using both the eulerian c-coverings $\mathcal{C}_{1}$ and $\mathcal{C}_{2}$. Nevertheless, in order to cover the simplicial vertex $a$ by exactly two cliques of a set of cliques $\mathcal{C}$, as required by Corollary 2 a, we need both $\{a, b\}$ and $\{a, c\}$ to be in $\mathcal{C}$. The same reason gives $\{d, b\}$ and $\{d, c\}$ in $\mathcal{C}$. Hence the edge $\{b, c\}$ must be covered by some extra clique of $\mathcal{C}$, and thus both $b$ and $c$ are in three cliques, contradicting the condition "every vertex is contained in exactly 2 cliques" of Corollary 2a. The result generalizes easily to infinite classes of graphs. For instance, every graph obtained by adding simplicial vertices to $G$ which are neighborhood-equivalent to the vertex $a$ cannot be proved hamiltonian by Corollary $2 \mathrm{a}$ but can be using Corollary 2.

The second graph $H$ of Fig. 3, despite its simplicity and the fact that it has pairwise-joint c-coverings, for instance $\{a b e c, d b e c\}$, cannot be proved hamiltonian using the results above. Indeed, the reader can check easily that the second condition of eulerian c-covering (Definition 5), said the "evenly-joint" condition, is not filled by any of the pairwise-joint c-coverings of $H$. Note that it could also be shown using a general result (not stated here) which implies in particular that $\{a b e c, d b e c\}$ is essentially the unique pairwise-joint c-covering of $H$. We resume the discussion concerning this example in the conclusion below.

\section{Conclusion and future works}

In Definition 6, the fact that the c-covering $\mathcal{C}$ is pairwise-joint ensures that $G \backslash \mathcal{C}$ is a multigraph. Then, the "evenly-joint" additional property of eulerian c-covering ensures the eulerianity of $G \backslash \mathcal{C}$ and thus the hamiltonicity of $G$ (cf. Theorem 1).
In Fig. 3 we give two hamiltonian graphs $G$ and $H$. The graph $G$ can be proved hamiltonian using the sufficient condition of Corollary 2 while, despite its similarity with the first one, the graph $H$ cannot, the reason being that the "evenly-joint" condition of Definition 5 fails. In order to obtain a provable hamiltonian graph $H^{\prime}$ from $H$ using Corollary 2, it is sufficient to add or remove a node in the intersection of the two cliques abec and $d b e c$, that is, to add or remove a vertex which is neighborhood-equivalent to the vertex $b$. Obviously in both cases the graph $H^{\prime}$ is hamiltonian iff $H$ is. Thus, a first direction for future researches would be to try to generalize this remark, that is, to find some general transformation of pairwise-joint c-coverable graphs into eulerian c-coverable graphs, transformation which preserves hamiltonicity of graphs. This transformation may then be used to get a broader notion of eulerian c-coverings in order to generalize Corollary 2.

A second direction of research concerns the design of an efficient algorithm able to find an eulerian c-covering of a graph iff such a c-covering exists. In [5], an efficient algorithm able to find a c-covering having the properties required by Corollary 2a is given. The soundness and completeness of this algorithm follow from a lemma stating that every clique of order at least four is necessarily a clique of such a c-covering. The lemma is not verified by eulerian c-covering and thus, in many cases, the algorithm answers "No" despite the existence of a eulerian c-covering. Nevertheless, at least in the case where the graph contains a simplicial vertex, it seems possible to design a new algorithm able to find an eulerian c-covering iff it exists. The possibility to obtain an algorithm working for every graph, containing a simplicial vertex or not, is currently under investigation.

Despite the apparent difference in nature between the sufficient conditions for hamiltonicity described in this article and the more usual ones found in literature, it may also be interesting to explore the possible links between them.

\section{References}

[1] D. Bauer, D. Broersma, E. Schmeichel, Toughness in graphs - A survey, Graphs and Combinatorics 22 (1) (2006) 1-35.

[2] M. DeLeon, A study of sufficient conditions for Hamiltonian cycles, Rose-Hulman Undergraduate Mathematics Journal 1 (1) (2000) (paper 4).

[3] G.A. Dirac, Some theorems of abstract graphs, Proc. London Math. Soc. 2 (1952) 69-81.

[4] R. Faudree, E. Flandrin, Z. Ryjàcek, Claw-free graphs - A survey, Discrete Mathematics 164 (1997) 87-147.

[5] S. Goodman, S.T. Hedetniemi, Sufficient conditions for graph to be Hamiltonian, Journal of Combinatorial Theory (B) 16 (1974) 175-180.

[6] R.J. Gould, Updating the Hamiltonian problem - A survey, Journal of Graph Theory 15 (1991) 121-157.

[7] R.J. Gould, Advances on the Hamiltonian problem - A survey, Graphs and Combinatorics 19 (2003) 7-52. 